\title{
Planification d'une étude expérimentale et rédaction d'une demande de subvention en 8 étapes et 28 questions
}

Georges BORDACE**, Beth DAWSON***

M essages clés - La planification d'un projet de recherche et la rédaction d'une demande de subvention sont des tâches complexes - La formulation de la question spécifique de recherche, qui fait suite au développement d'une problématiquederecherche, est l'étapecrucialedu processus. Elles'appuiesur une analysecritiquedes référen ces scientifiques, qui permet de dégager un cadre conceptuel. - Le choix des instruments de mesure et celui du plan de la recherche dépendent de la question de recherche. Leur pertinence doit être argumentée et leur limites discutées. - La détermination des échantillons d'étude et de leur taille a intérêt à s'appuyer sur une démarche statistique préalable. Le choix des outils stati stiques utili sés pour étayer l'interprétation des résultats doit être pertinent et justifié. - L'échéancier, la distribution des rôles et le budget de la recherche doi vent être définies avec précision. - L e dossier de demande de subvention doit chercher à convaincre «l'organisme subventionnaire » que la problématique de recherche proposée est pertinente par rapport à ses missions, que l'étude présentée est scientifiquement valide et défendable sur le plan de l'éthique, que les auteurs du projet ont la capacité scientifique et les ressources suffisantes de le conduire à terme et que le budget prévu est crédible.

Mots dés Planification ; étude expérimentale; demande de subvention.

Key learning points • Preparing a study and writing a research grant proposal are complex undertakings • The research question and study design are the two most important components of a sudy. They rely on a critical analysis of the literature and a clear conceptual framework and problem statement. - 0 utcomes of interest dictate instrumentation, not the other way around. - Sampling method and sample size must be justified, preferably based on a judicious selection of statistical analyses. - The study schedule, personnel, and budget must be presented clearly - Funding agencies look for studies that are important and relevant to their mission, scientifically sound, conducted by capable researchers, and reasonably priced.

Keywords Study design; experimental research; grant writing.

Pédagogi e M édicale 2003 ; 4 : 103-14

\section{Introduction}

La planification d'un projet de recherche et la rédaction d'une demande de subvention sont des tâches complexes, non seulement à cause du grand nombre d'éléments à considérer mais aussi parce que chaque élément joue un rôle crucial dans lesuccès de l'entreprise. Par exemple, un projet de recherche peut porter sur une question pertinente mais si la méthode d'échantillonnage est biaisée, la valeur de l'étude peut être sérieusement compromise, au

\footnotetext{
* Traduit par G Bordage, avec l'autorisation de Medical Education, de « Experimental study design and grant writing in eight steps and 28 questions "'

** Department of Medical Education - College of Medicine - University of Illinois at Chicago - Etats-Unis.

** * Department of Medicine - College of Medicine - Southern Illinois University - Springfield, IL - Eats-Unis.

Correspondance: Georges Bordage - Department of Medical Education - College of Medicine - University of Illinois at Chicago 808 South Wood (M/C 591) - Chicago, IL $60612-7309$ - Etats-Unis - Tél. : 001 (312) 9967349 - Fax : 001 (312) 4132048 mailto:bordage@uic.edu
} 


\section{Références}

point d'en invalider les résultats. Ainsi, nous proposons une démarche en 8 étapes et 28 questions afin defaciliter la planification d'une étude expérimentale et la rédaction d'une demande de subvention. Cette démarche permettra de garder en tête tous les éléments essentiels du processus et d'assurer ainsi la qualité du protocole de recherche et de la demande de subvention. L esétapes et les questions sont résumées dans le Tableau 1. Ce tableau peut être utilisé comme une liste de vérification afin de s'assurer que tous les éléments ont été considérés. Chaque étape sera discutée et des exemples seront présentés.

G. B ordage a récemment analysé les commentaires des éval uateurs externes au moment de recommander l'acceptation ou le rejet de manuscrits soumis pour publication concernant les travaux en pédagogie médicale ${ }^{2}$. U ne attention particulière sera portée sur les forces et les faiblesses notées par les évaluateurs externes concernant la planification d'un projet de recherche et la rédaction scientifique. Presque la moitié des 19 critères principaux donnés par les évaluateurs externes doivent être pris en compte dès la planification d'un projet de recherche si les chercheurs veulent éviter des surprises désagréables au moment de soumettre leurs résultats pour publication. Ces critères, énoncés de façon positive, sont les suivants : le sujet de recherche est important ; l'introduction contient un énoncé clair du problème et une analyse critique et actualisée des références ; le plan de recherche (type d'étude) et les instruments de mesure sont optimaux ; l'étude contient un échantillon adéquat et non biaisé ; les analyses statistiques sont appropriées et optimales. Bien que la démarche proposée s’applique directement à des études de type expérimental, la plupart des principes présentés s'appliquent aussi à d'autres types de travaux scientifiques tels que des études d'observation ou d'évaluation ou encore des études qualitatives.

\section{La planification d'une étude experimentale \\ Étapel : Q uestion de recherche}

1- Q uel sujet de recherche ou problème vous intéresse?

2- Q uelles recherches ont déjà été effectuées à ce sujet ?

3- Q uels sont les effets [variable (s) dépendant (s)] qui vous intéressent principalement?

4- Q uelle intervention (variable indépendante) vousinté resse?

5- Recherchez-vous des différences ou une association entre variables?

6- A quel groupe (population) désirez-vous appliquer vos résultats?
7- Q uelle est votre question de recherche?

8- Quelle réponse attendez-vous?

Les hypothèses de recherche.

9- Pourquoi cette question est-elle importante?

La pertinence.

L'élément le plus important d'un projet de recherche est la question de recherche $e^{3}$ qui est la pierre angulaire de toute I'entreprise. D'emblée, les chercheurs font face à deux défis en voulant planifier une nouvelle étude : choisir un sujet de recherche important et formuler une question de recherche pertinente. Le critère principal pris en compte par les évaluateurs externes pour recommander l'acceptation d'un manuscrit scientifique est la formulation d'une question de recherche importante et opportune. La pertinence d'une question de rechercheva bien au-delà du fait que la question correspond à la mission et aux priorités de l'organisme subventionnaire*, bien qu'il sagisse là d'impératifs importants à ne pas ignorer. Les chercheurs doivent aussi démontrer de façon convaincante que l'étude proposée représente un pas en avant pour le domaine et que les résultats anticipés contribueront à l'avancement des connaissances, autant théoriques ou méthodologiques que pratiques.

La construction d'une problématique de recherche constitue le cheminement intellectuel argumenté par lequel les chercheurs, à partir d'un problème général (écart entre une situation constatée et une situation souhaitée), identifient successivement une question générale (que nous dit la recherche sur cesujet ?) puis un problèmespécifique (en quoi les recherches antérieures disponibles n'ont-elles pas résolu le problème?) et enfin une question spécifique de recherche (quelle contribution personnelle les chercheurs se proposent-ils d'apporter au problème de recherche ?). Cette démarche implique une analyse critique, bien centrée et bien réfléchie, des références scientifiques disponibles sur le sujet. Cette analyse sera exploitée d'une part, pour présenter un état de la question probant et convaincant ; elle permettra d'autre part, aux chercheurs d'identifier et de rendre explicite le cadre conceptud (ensemble articulé de théories, de modèles, de concepts, de lois ou d'indicateurs) qu'ils utilisent pour formuler leur question de recherche et pour, ultérieure ment, interpréter leurs résultats. D e la rigueur et de la qualité de cette analyse dépend fondamentalement la solidité des bases del'étude. L'énoncédu problème et celui de la question de recherche ne doivent être ni trop restreints ni trop larges ou trop flous. D es questions simplistes risquent de mener à des réponses triviales, alors que des ques tions trop larges ne seront probablement pas faciles à étudier. Considérez, par exemple, la question de recherche 
suivante avec ses éléments de base (notés entre parenthèses). Est-ce que les étudiants, dont les professeurs de sciences de base en médecine (population visée) ont suivi des ateliers de perfectionnement pédagogique (intervention : premier niveau de la variable indépendante) pour dével opper leurs compétences de présentateur interactif lors de leçons magistrales, auront de meilleures (nature de la relation entre variable: différence entre deux niveaux de la variableindépendante) notes lors des examens de fin de semestre (mesure des résul tats: variable dépendante) que les étudiants dont les professeurs ont été formé à l'ai de de démonstrations vidéos (intervention : deuxième niveau de la variable indépendante) ? Le cadre conceptuel utilisélors de la formulation de cette question vient de théories de l'apprentissage et de l'enseignement selon lesquelles les étudiants apprennent mieux lorsqu'ils sont activement impliqués dans le processus d'enseignement-apprentis sage. D es quatre éléments de la question de recherche (1) variable indépendante (celle manipulée directement par le chercheur), (2) variable(s) dépendante(s) (celle(s) dont on observe les variations résultant del'intervention), (3) relation entre les variables et (4) population visée - les deux derniers sont le plus souvent laissés flous ou mal définis.

La nature de la relation entre les variables est pourtant essentielle pour déterminer les analyses des données les plus optimales et pour tirer des conclusions appropriées. Est-ce que le but de l'étude est de comparer la performance des étudiants selon que leurs professeurs ont été exposés à différentes modalités de perfectionnement pédagogique (par exemple respectivement des ateliers ou des démonstrations vidéos), ce qui revient à chercher une différence? 0 u bien s'agit-il de déterminer le niveau d'association entre, par exemple, le nombre d'interactions enseignant-étu diants et l a performance des étudiants? De plus, les variables dépendantes (résultats) doivent être suffisamment opérationnelles pour être observées ou mesurées. D es concepts généraux (par exemple, techniques de présentation) sont trop vagues et risquent de prêter à confusion. Les chercheurs doivent définir les aspects précis du concept qui les intéresse. Par exemple, dans le cas des techniques de présentation lors de leçons magistrales, s'agit-il de la qualité des objectifs d'apprentissage, des compétences de communication du présentateur, de la participation de l'auditoire, de la qualité du matériel audiovisuel ou de la satisfaction des étudiants ? L a nature précise de la variable dépendante et le type de relation entre les variables indépendante et dépendante détermineront letype d'analyse des données et lesconclusionsqui peuvent en être tirées.
D 'autre part, à qui les chercheurs désirent-ils appliquer (généraliser) leurs résultats ? Les chercheurs doivent bien différencier d'une part, la population visée, c'est-à-dire ceux à qui ils veulent généraliser leurs résultats, et d'autre part, l'échantillon, c'est-à-dire ceux qui ont participé à l'étude. Bon nombre d'études en éducation sont menées avec des échantillons de circonstance (à la portée de la main) ou avec des groupes statiques qui peuvent être peu représentatifs de la population (pauvre validité externe) et qui risquent ainsi delivrer des résultats peu généralisables. Les chercheurs doivent garder à l'esprit la généralisabilité de leurs résul tats lorsqu'ils choisissent les participants pour l'étude et justifier leur échantillon et leur méthode d'échantillonnage aux évaluateurs du projet (et aux lecteurs éventuels).

La présentation de variables opérationnelles, et de leur interrelation, ainsi que la définition de la population contenue dans la question conditionnent directement la formulation d'hypothèses de recherche, c'est-à-dire des résultats anticipés. Par exemple, l'hypothèse descriptive (celle préférée par les chercheurs) peut être : «II est postulé que les étudiants dont les professeurs ont suivi des ateliers de perfectionnement pédagogique auront de meilleures notes lors des examens de fins de semestre que les étudiants dont les professeurs ont été formé àl'aide de démonstrations vidéos. » $\mathrm{N}$ on seulement les hypothèses de recherche guideront le type d'étude et d'analyse des données mais elles serviront égal ement à alerter les chercheurs quant à la présence possible de variables confondantes (toute explication alternative) qui devront être contrôlées dans le plan de recherche. L'importance de la question de recherche et de chacun de ses éléments constituants ne peut pas être sous-estimée. Tout dépend dela question de recherche, d'où son importance cruciale. Ainsi, la question de recherche doit être énoncée tôt dans la demande de subvention, éventuellement dès la première phrase ou lepremier paragraphe, et doit être décrite et justifiée en détail. Rien dans la question de recherche ne doit donner matière à interprétation. D es erreurs d'omission à cette étape initiale de la planification peuvent entraîner des conséquences graves et coûteuses plus tard.

\section{Étape 2 : Instruments de mesure}

10- U tiliserez-vous un instrument de mesure existant, que vous allez modifier ou non, ou en développerez-vous un nouveau?

11- Q uelles sont les qualités psychométriques des mesures (scores) ?

NDLR : «O rganisme subventionnaire » : pour désigner les organismes qui subventionnent la recherche. Ce terme semploie dans les pays anglo-américains et surtout au Q uébec. 


\section{Références}

Une fois que les variables dépendantes sont clairement établies et définies, les chercheurs doivent maintenant choisir un ou des instrument(s) de mesure approprié(s) afin de bien mesurer les résultats qui les intéressent. U ne erreur fréquenteà cette étape est de choisir un instrument simplement parce qu'il est facilement accessible plutôt que de dével opper un instrument plus approprié. C'est le cas, par exemple, lorsque des chercheurs choisissent d'utiliser des notes provenant d'un examen écrit national sur les sciences du comportement pour mesurer la capacitéde communiquer avec des malades lors de situations cliniques difficiles plutôt que de développer un instrument de mesure plus approprié, tel qu'une liste de vérification avec des malades standardisés. La nature de la variable dépendante doit dicter le choix des instruments et non l'inverse.

Ainsi, alors que certains instruments sont d'emblée disponibles, d'autres devront être modifiés ou développés denovo. L'utilisation d'instruments existants est préférable autant que possible lorsque leurs qualités psychométriques (par exemple, fidélité et validité) en ont déjà été établies. II sera ainsi possible de comparer les résultats de la présente étude à des résultats antérieurs. Si les instruments doivent être modifiés ou développés, les qualités psychométriques des scores qui seront obtenus lors de l'étude doivent être discutées, ainsi que toutes leurs limitations ou imperfections. Les modalités d'administration, les échelles de mesure (c'est-à-dire nominale, ordinale, à intervalle ou ratio) et les méthodes d'attribution des scores doivent être clairement présentées afin de pouvoir déterminer la validité des mesures et l'interprétation des résultats à venir.

\section{Étape 3 : Plan de recherche}

12- Voulez-vous intervenir ou simplement observer?

13- Avez-vous besoin d'un groupe contrôle?

14- Comment allez-vous contrôler les variables confondantes?

15- Q uel est le meilleur plan de recherche pour répondre à la question?

Le deuxième élément le plus important d'une étude est le plan de recherche, c'est-à-dire les moyens que les chercheurs entendent mettre en œuvre pour répondre à la question. Le choix d'un plan de recherche et le contrôle des variables confondantes potentielles représentent deux autres défis de taille pour les chercheurs. La recherche en éducation est souvent menée dans des situations naturelles qui risquent de menacer la validité de l'étude, en raison par exemple d'une perte de participants, de biais de sélection, de la présence d'événements historiques ou de la maturation des participants. Frankel et Wallen ${ }^{4}$ ont éta- bli une typologie des plans de recherche qual ifiés respectivement de "expérimentaux faibles », " expérimentaux véritables » et « quasi-expérimentaux » selon le niveau de contrôle des facteurs invalidants (validité interne). Les plans expérimentaux «faibles » comprennent les plans de recherche à cas unique, les plans à un groupe avec pré-test et post-test et les plans avec comparai son de groupes existants. Les plans expérimentaux « véritables » (avec randomisation des différentes interventions) comprennent les plans à groupes indépen dants avec groupetémoin et posttest seulement ou avec prétest et post-test, et assignation al éatoire avec appariement. Les plans « quasi-expérimentaux » (absence d'assignation aléatoire des différentes interventions) comprennent des plans avec appariement seulement, les plans contrebalancés, et les plans à séries temporelles. Les chercheurs doivent présenter une argumentation convaincante quant au choix de leur plan de recherche, compte tenu de leur situation particulière et notamment de leurs contraintes, et quant aux mesures prises pour contrôler les variables confondantes potentielles, soit par le type de plan de recherche (par exemple, un plan avec groupes contrebalancés dans le cas d'interventions ou de stimulus multiples), soit par des analyses statistiques post hoc (par exemple, des analyses avec mesures répétées ou une analyse de covariance), soit en utilisant les deux méthodes. Les chercheurs peuvent ajouter du poids à leur choix en présentant deux ou trois plans de recherche alternatifs et en démontrant pourquoi tel plan est supérieur aux autres, compte tenu des circonstances particulières de l'étude proposée.

\section{Étape-4 : Statistiques}

16- Q uelle méthode statistique est optimale?

Le critère principal pris en compte par les évaluateurs externes pour recommander le rejet d'un manuscrit pour publication est l'utilisation d'analyses statistiques inappropriées, sous-optimales ou incomplètes. Afin de choisir les statistiques appropriées, les chercheurs doivent prendre en considération plusieurs facteurs, à savoir le nombre et la nature des variables, la nature de la relation entre les variables (telle qu'exprimée dans les hypothèses de recherche) et le plan de recherche. ${ }^{5,6}$ L'utilisation de $^{\prime}$ méthodes statistiques simples et appropriées aboutit le plus souvent à des conclusions claires et franches. L'accès facile à des logiciels statistiques peut mener des novices (ou des statisticiens amateurs) à choisir des statistiques inappropriées ou à les utiliser de façon inappropriée. La consultation précoce d'un statisticien peut s'avérer être un excellent investissement.

C'est à cette étape que les chercheurs peuvent considérer si certaines variables confondantes potentielles, qui ne 
peuvent pas être contrôlées par le choix d'un plan de recherche, peuvent l'être par des analyses statistiques particulières, par exemple en effectuant des analyses de covariance ou des analyses de régression multiple ou logistique. D es analyses statistiques bien réfléchies et parfois innovatrices ont été particulièrement louées par les évaluateurs externes.

\section{Étape 5 : Échantillon et méthode d'échantillonnage}

17- Q uels sont les critères d'inclusion et d'exclusion des participants?

18- Comment allez-vous obtenir les participants?

19- Comment allez-vous assigner les participants à leurs groupes?

20- Combien de participants sont nécessai res? Puissance statistique.

La rigueur des méthodes d'échantillonnage, des critères d'inclusion et d'exclusion des participants et d'assignation des participants aux différentes interventions, ou au groupe contrôle, est un aspect critique d'une étude, qui permet d'éviter la présence de biais. De plus, la détermination du nombre de participants (conditionnée par un calcul de puissance statistique ou une analyse de tolé rance) est cruciale, non seulement afin quelesconclusions tirées des résultats soient valides, mais aussi afin de maximiser les ressources. L'utilisation de trop peu de participants peut mener à des conclusions erronées, alors que I'utilisation de trop de participants peut mener à des conclusions triviales ou au gaspillage de ressources limitées. Ce dernier point est particulièrement cher aux organismes subventionnaires.

II y a plusieurs excellents logiciels disponibles sur le marché qui permettent de calculer la taille de l'échantillon nécessaire; certains sont des logiciels spécifiques (par exemple, nQ uery ${ }^{T M}$ ), d'autres font partie d'un logiciel statistique intégré ( $p$ ar exemple, SPSS ${ }^{\mathrm{TM}}$, $N$ CSS $^{T M}$, Epilnfo ${ }^{T M}$ ). Encore une fois, il est particuliè rement sage de consulter un statisticien pour qu'il aide à déterminer le nombre de participants nécessaire. Q uatre données sont nécessaires pour déterminer la taille de l'échantillon : (1) le niveau de confiance désiré (1- $\alpha$, habituellement .95 ou .99, où al pha est la probabilité de conclure à tort qu'une différence existe alors qu'elle est liée au hasard et non à l'intervention), (2) le niveau de puissance (1- $\beta$, habituellement .80, c'est-àdirela probabilité de détecter une différencesi une telle différence existe réellement), (3) la plus petite différence pratiquement significative (il s'agit d'un juge- ment ; par exemple : Q uelle amélioration devront démontrer les notes des étudiants pour que le doyen décide de recommander des ateliers de formation ?), et (4) une estimation de la variance (écart-type estimé à partir de références antérieures ou de résultats préliminaires provenant d'essais pilotes). Enfin, il est aussi prudent d'ajouter des participants additionnels au calcul de la taille de l'échantillon (sur-échantillonnage) en anticipation d'une perte éventuelle de participants. Les évaluateurs externes ont loué l'utilisation d'échantillons appropriés et en nombre adéquat.

\section{Étape 6 : Recueil des données et contrôles de qualité}

21- Comment allez-vous recueillir les données et contrôler leur qualité?

La saisie des données et leurs contrôles de qualité doivent être présentés avec suffisamment de détails pour que les évaluateurs soient convaincus que l'intégrité de I'étude sera maintenue. U ne attention particulière doit être portée à I'entraînement des observateurs et au dépistage précoce de biais possibles qui pourraient apparaître pendant l'étude, comme la perte de participants ou des biais de sélection. D es contrôles de qualité doivent rester en place tout au long du recueil et des analyses des données. Les tâches et le personnel mandaté pour ces tâches doivent être décrits clairement.

\section{Étape-7 : Échéanciers et budget}

22- Q uels seront les échéanciers ? L es horaires.

23- Q ui fera quoi ? Le personnel.

24- D e quel équipement, de quel matériel aurez-vous besoin?

25- Q uels seront les coûts ? Le budget.

Q ui fera quoi et quand ? Q uel équipement sera nécessaire pour mener l'étude et quels seront les coûts ? La quantité de détails à fournir dépendra des instructions données par l'organisme subventionnaire. Par exemple, certains organismes exigent des budgets détaillés alors que d'autres ne veul ent que des budgets globaux. Q uelles que soient les instructions, il est toujours utile d'organiser les échéanciers et le budget sel on cinq grandes phases d'une étude : (1) préparation initiale (la mise en route du projet), (2) les essais pilotes des instruments de mesure et des procédures de recueil de données, (3) le recueil des données, (4) l'analyse et l'interprétation des données et (5) la diffusion des résultats, y compris la préparation d'un rapport pour l'organisme subventionnaire et de manuscrits pour publication 


\section{Références}

scientifique et présentation à des congrès. Pour chaque phase, les activités peuvent être présentées dans un tableau contenant quatre colonnes indiquant (1) la date (quand), (2) les tâches (quoi), (3) le personnel (qui) et (4) la durée (combien de temps) de chaque activité.

\section{Étape 8 : Protocole de recherche et demande de subvention}

26- Comment allez-vous suivre les progrès del'étude? Le protocole.

27- Q uelles sont les priorités de l'organisme subventionnaire?

28- Q uels formulaires devrez-vous compléter et quelles procédures suivre?

Ayant tous les ééments de l'étude en main, il est mainte nant temps de rédiger le protocole de recherche et la demande de subvention. Bien que le protocole et la demande partagent beaucoup d'éléments en commun, ils sont quelque peu différents. La demande de subvention sadresse à l'organisme subventionnaire afin d'obtenir des fonds. La demande a pour but de promouvoir l'étude et de convaincre les éval uateurs et l'organisme subventionnaire de subventionner le projet de recherche al ors que le protocole est davantage procédural. Ce dernier contient les instructions et les procédures détaillées à suivre tout au long du projet et représente un aide mémoire des tâchesà accomplir. Ceci permet d'éviter aux chercheurs, pour paraphraser $\mathrm{M}$ ager ${ }^{7}$ à propos des objectifs d'apprentissage, de se retrouver dans une situation différente que celle qu'ils avaient prévue, de plus sans vraiment en être conscients!

Les organismes subventionnaires posent typiquement quatre questions au moment de décider si le projet sera subventionné ou non. (1) Est-ce que le sujet (la question de recherche) est pertinent compte tenu de la mission de I'organisme? La réponseà cette question se trouve dans le titre, le résumé et l'énoncé du problème. (2) Est-ce que l'étude est scientifiquement valide? La réponse se trouve dans la question et les hypothèses de recherche, ainsi que dans le plan de recherche et la taille de l'échantillon. L'organisme s'assurera aussi que le projet a été approuvé par le comité d'éthique de l'institution où réside les chercheurs. (3) Est-ce que les chercheurs sont capables de mener à terme le projet proposé ? La réponse se trouve dans leur formation et leur expérience, ainsi que dans l'importance du soutien institutionnel et l'envergure du projet. (4) Combien coûtera le projet ? Ces informations se trouvent dans le budget et les échéanciers. Lorsqu'un comitééval ue une demande de subvention, seuls deux ou trois membres liront la demande au complet. Les autres membres se fieront principalement aux informations contenues dans le titre, le résumé, le budget, les échéanciers et aux recommandations des évaluateurs externes, d'où l'importance du résumé ; point qui sera discuté davantage plus loin.

Les critères d'éval uation utilisés par les évaluateurs d'une demande de subvention sont en général les mêmes que ceux utilisés pour évaluer un manuscrit scientifique soumis pour publication (voir Bordage?2, ainsi queles critères d'évaluation proposés dans les rapports de groupes de travail mandatés par certaines revues telles que Academic M edicine ${ }^{8}$ ou The British M edical J ournal ${ }^{9}$ ). Enfin, la décision de subventionner un projet de recherche est souvent effectuée en deux temps. Tout d'abord les membres du comité de sélection jugent la qualité scientifique de la demande, pour ensuite, dans un deuxième temps, décider du budget à accorder. Ainsi le mérite scientifique de l'étude proposée est généralement jugé de façon indépendante du coût du projet.

\section{La rédaction d'une demande de subvention}

Le type d'information et le niveau de détail exigés dans une demande de subvention varient selon les organismes subventionnaires. Les chercheurs doivent porter une attention particulière aux exigences spécifiques de I'organisme subventionnaire, décrites en détail dans les instructions aux chercheurs, faute de quoi ces derniers risquent de voir leur demande retournée pour ne pas avoir suivi les règles exigées. II est important de ne pas semer la confusion chez les évaluateurs en utilisant des formats ou contenus non orthodoxes. En revanche, un résumé et une demande qui sont rédigés selon les règles impressionneront favorablement les évaluateurs. Un guide générique pour la préparation d'une demande de subvention (préparé, avec permission, en partie selon les formulaires des N ational Institutes of $\mathrm{H}$ ealth ${ }^{10}(\mathrm{~N} I \mathrm{H})$ ) est présenté dans le Tableau 2 ; il consiste en 10 points principaux : page titre, résumé, personnel principal, budget pour la première année, budget pour la période restante, justification du budget, ressources, plan de recherche, références citées et approbation éthique. LesTableaux 1 et 2 peuvent être utilisés comme listes de vérification afin de s'assurer que tous les éléments exigés sont présents, tant pour l'étude que pour la demande de subvention. Les détails de l'étude sont particulièrement bien maîtrisés par les chercheurs au moment où ils rédigent la demande de subvention. Ainsi, une demande bien préparée constitue aussi un bon investissement pour la rédaction du manuscrit (publication) à venir. 


\section{Le titre}

Le premier élément de la demande de subvention, son titre, représente un moyen important de marketing de l'étude proposé. Le titre lance un message clair aux lecteurs quant au sujet principal de l'étude et aux intentions des chercheurs. Les mots clés contenus dans le titre doivent plaire d'emblée aux évaluateurs et doivent correspondre expressément à la mission de l'organisme subventionnaire. Le titre constitue le résumé le plus court possible de l'étude, où à la fois le sujet et son importance sont mis en exergue. C'est ce à quoi répondent respectivement les composantes indicative (le quoi) et informative (le message) du titre (voir H uth ${ }^{11}$ et Bordage ${ }^{12}$ ). Par exemple : présentation interactive lors de leçons magistrales en sciences de base : ateliers de formation plus efficaces que démonstrations vidéos ? Letitre doit capter l'attention de l'organismesubventionnaireet desévaluateurs, tout en demeurant fidèle à l'étude. U $\mathrm{n}$ des critères principaux pris en compte par les évaluateurs externes lors du rejet de manuscrits pour publication est la présence de titres trompeurs. Les chercheurs devront démontrer clairement qu'ils peuvent « livrer la marchandise » annoncée dans le titre.

\section{Le résumé}

L'importance du résumé a été mentionnée précédemment, surtout parce qu'il sagit d'un des seuls éléments d'une demande qui est lu par tous ceux qui auront à décider du sort de la demande. Un résuméstructurépeut servir à la fois d'éément organisateur et d'aide-mémoire. ${ }^{13,14}$ Les composantes du résuméstructuré sont : les objectifs, le contexte et la pertinence, le plan de recherche, la population visée et échantillonnée, les mesures des résultats, les résultats et les conclusions anticipés. La section des résultats et des conclusions est utilisée dans la demande pour annoncer les résultats anticipés (les hypothèses descriptives) et pour promouvoir leur importance pour l'organisme subventionnaire, l'institution d'où émanent les chercheurs et pour le domaine en général.

\section{Personnel principal}

La rubrique du personnel contient des informations pré cises et concises à propos du chercheur principal et des autres personnes impliquées dans l'étude, y compris les co-chercheurs, les assistants de recherche, le coordinateur du projet, le personnel de support et les consultants. Les organismes subventionnaires ne veulent pas de long curriculum vitae, plein de détails. Une courte autobiographie de une ou deux pages est généralement suffisante. Les évaluateurs veulent glaner et évaluer rapidement les informations. Ils recherchent des données clés concernant la compétence et l'expérience des chercheurs. Ils veulent connaître la scolarité de chacun(e), leur formation en recherche, leur poste, le nombre de subventions obtenues dans le passé et le nombre de publications revues par des pairs (y compris une courte liste de leurs références pertinentes par rapport à l'étude proposée). C es informations seront utilisées pour évaluer si les chercheurs sont capables de mener à termel'étude proposée de façon satisfaisante, autant sur le plan scientifique qu'administratif.

\section{Budget}

Le budget est présenté dans trois sections différentes : le budget pour la première année de subvention, le budget pour la période restante et la justification du budget. En séparant la première année du reste du projet, l'organisme subventionnaire peut déterminer rapidement les allocations budgétai res pour l'année fiscale à venir. Alors que certains organismes exigent des budgets détaillés, d'autres n'exigent que des budgets globaux. Q uelles que soient les exigences des organismes, il sera toujours utile pour les chercheurs de préparer un budget détaillé afin d'estimer d'une part les coûts lors de la planification del'étude et de suivre d'autre part les dépenses mensuelles une fois le projet en marche.

Les items budgétai res comprennent : le personnel, les consultants, l'équipement, le matériel de bureau (papete rie), le coût des transports reliés au projet et à la présenta tion des résultats à des congrès, les dépenses diverses (planifier les dépenses inattendues) et les frais administratifs. C ertains organismes subventionnaires limitent les types de dépenses permises. Par exemple, certains ne permettent pas les frais administratifs ou imposent des limites (par exemple, 10 \% maximum des coûts directs), alors que d'autres restreignent l'achat d'équipement comme celui d'ordinateurs. L'achat d'ordinateurs portables pour la saisie des données peut êtrejustifiableà l'occasion. II est important de suivre à la lettre les règles et les procédures del'organismesubventionnaire. Enfin, si lebudget savère trop dispendieux, considérez la possibilité de scinder le projet en phases successives (sous-projets) avec des budgets distincts qui pourront être versés de façon séquentielle suite à des rapports d'étapes intermédiaires.

\section{Ressources}

La section des ressources comprend les besoins en équipe ment et en locaux pour bien mener la recherche, à partir de ressources existantes (contribution en nature) ou à inclure dans le budget soumis. Les items à considérer incluent les locaux, l'équipement informatique (ordinateurs, scanner, imprimantes, logiciels) et l'analyse des données

Les chercheurs peuvent aussi inclure dans cette section 


\section{Références}

des lettres de soutien du projet provenant des autorités institutionnelles comme le doyen, les chefs de département ou les directeurs de programme. Chacun peut faire valoir la pertinence du projet et l'apport particulier de l'étude pour eux et pour le domaine, ainsi que leur désir de participer. Les chercheurs peuvent préparer des brouillons de lettre afin de faciliter la tâche de ceux qui auront à soumettre des lettres de soutien. Les lettres ellesmêmes peuvent être annoncées dans cette section et incluses dans une annexe à la fin de la demande de subvention.

\section{Plan de recherche}

Le plan de recherche représente le cœur de la demande. Le plan s'étend de l'énoncé du problème aux échéanciers et horaires, en passant par la question de recherche et la cueillette et l'analyse des données. Les sous-sections sont les mêmes que celles du résumé structuré. Il est important de suivre une structure parallèle entre les différentes sections de la demande ; par exemple, les sous-sections du résumé et du plan doivent être les mêmes et les objectifs et hypothèses doivent toujours être présentés dans le même ordre.

Ceci facilitera grandement la lecture de la demande. La plupart des organismes subventionnaires imposent un nombre limité de pages pour cette section, en règle 25 pages maximum. Encore une fois, il est important de suivre les instructions à la lettre. Toute dérogation peut mener au rejet immédiat de la demande.

\section{Le style}

Un style simpleet clair plaira aux évaluateurs. Ils n'aiment pasnaviguer péniblement dansun fouillis de détails où ils doivent constamment deviner les intentions des chercheurs. La logique doit être transparente partout, et en particulier au moment de passer d'une section à l'autre. La terminologie doit être uniforme partout. Lorsque la terminologie varie, par exemple du mot «groupes » au mot «programmes », alors que les deux mots se réfèrent aux mêmes entités, le lecteur peut facilement devenir confus et frustré. Avant de soumettre la demande, utilisez un vérificateur informatique orthographique et grammatical et faites lire le brouillon final par un ou deux collègues qui ne sont pas familiers avec l'étude.

\section{Références citées}

L'avant-dernière section contient la liste des références citées. II ne s'agit pas d'une bibliographie ou de la liste de l'ensemble des références lues par les chercheurs. C ette section ne contient que les références clés citées dans la demande. L es chercheurs doivent démontrer qu'ils connaissent bien l'ensemble du domaine et ont su positionner leur étude de façon à produire des résultats éventuels qui seront probants et utiles.

II n'est pas nécessairement avantageux d'inclure beaucoup de références. U neanalyse critique et bien étayée des références aura plus de poids auprès des évaluateurs que la présentation d'une liste exhaustive, sans discernement. Les chercheurs doivent aussi garder à l'esprit que certains évaluateurs sont des experts en la matière et connaîtront les références de long en large.

\section{Éthique}

L'approbation éthique de l'étude proposée par les autorités institutionnelles devient de plus en plusla norme dans le domaine de la recherche en éducation et est requise par les organismes subventionnaires et les revues scientifiques ${ }^{15,16,17}$. Le but visé est de protéger les participants et leurs dossiers (données) en évaluant les risques et bénéfices de partici per à l'étude, les biais de sélection, la confidentialité et les incitatifs à participer à l'étude. Lorsqu'un consentement informé est exigé des participants, une copie du consentement doit être incluse en annexe de la demande de subvention. L es éléments principaux du consentement comprennent : les buts de l'étude (sans toutefois induire un effet expérimentateur), les risques et bénéfices, les alternatives à la participation, les garanties de confidentialité, les droits des participants et le nom des personnes à contacter pour tout renseignement additionnel ou pour se désister del'étude. Le plus souvent, la lettre attestant l'approbation éthique n'est pas disponible au moment de poster la demande de subvention. D ans ce cas, il convient simplement d'indiquer que l'approbation est en attente (voir item 1.4 de la page titre) et de s'engager à la fournir au moment où elle sera disponible. Attention, I'approbation éthique doit être obtenue avant de recueillir les données. Les comités d'éthique refuseront de sanctionner une étude de façon rétroactive.

Les mêmes principes éthiques qui empêchent la double soumission de manuscrits à plusieurs revues scientifiques sappliquent en cas de soumissions de demande de subvention. Toutefois, certains organismes permettent la double soumission lorsqu'ils en sont informés à l'avance. En cas d'acceptation du projet par deux organismes subventionnaires, un partage des coûts peut être négocié

\section{Organismes subventionnaires}

Les organismes subventionnaires peuvent être classés selon leur provenance géographique, c'est-à-dire locale, provinciale, nationale ou internationale. Les organismes locaux comprennent les agences institutionnelles ou civiques comme les programmes de subvention aux jeunes chercheurs, les fondations et fiducies, les pro- 
grammes de subvention au développement et les programmes de formation à la recherche pour les étudiants. Les agents des relations extérieures et du bureau des subventions et contrats de votre institution peuvent aider à repérer des fonds ou à identifier des réseaux de personnes ressources. Les organismes provinciaux ou régionaux incluent les collèges ou corporations responsables d'émettre les droits de pratique (surtout pour cequi a trait à des projets avec une composante d'évaluation) ou les associations ou sociétés professionnelles. Les agences nationales et internationales comprennent les gouvernements (ministères de la santé ou de l'éducation, les bureaux de la main d'œuvre ou les instituts de recherche), les fondations, les organismes professionnels (par exemple, les organismes nationaux responsables des examens permettant l'accès au droit de pratique ou les associations des facultés de médecine) et enfin les compagnies pharmaceutiques et les compagnies avec articles brevetés.

\section{Conclusion}

Les 8 étapes, 28 questions et 10 éléments ne sont jamais pris en compte de façon séquentielle. II s'agit plutôt d'un processus itératif où les chercheurs affinent petit à petit leur sujet de recherche et la question de recherche et soupèsent tour à tour les avantages et désavantages de certains plans de recherche et de certains instruments de mesure. Cette démarche mène souvent les chercheurs à reconsidérer des décisions prises et parfois à prendre de nouvelles orientations. Par exemple, un problème d'instrument de mesure peut forcer les chercheurs à redéfinir leurs variables dépendantes ou encore les exigences de taille d'échantillon peuvent mener à exclure certaines interventions, faute de participants disponibles. Plus il y aura réflexion et discussion au point de départ, pendant la phase de planification, meilleures seront les chances d'éviter des circonstances désagréables plus tard ou, pire encore, la survenue d'erreurs irrémédiables.

Alors que la rédaction de la demande de subvention peut ne prendre qu'une ou deux semaines, la planification détaillée et bien réfléchiedu projet prendra beaucoup plus de temps. II n'est ainsi pas déraisonnable de se mettre à l'ouvrage 4 à 6 mois avant la date limite. Aucune étude n'est parfaite. Les limites de l'étude, liées par exemple au caractère sous-optimal du plan de recherche, à la présence de certaines variables confondantes, ou à des instruments de mesure imparfaits, doivent être discutées tôt dans la démarche afin d'éviter un rejet par l'organisme subventionnaire, ou pire encore, un rejet au moment de la soumission pour publication des résultats. En guise de pré vention, I'utilisation d'une démarche systématique et détaillée, telle que celle constituée par les 8 étapes et les 28 questions présentées ici, ai dera à limiter les risques et à augmenter leschances de succès, autant pour les chercheurs que pour la communauté scientifique en général.

Remerciements: N ous sommes grandement reconnaissants envers le professeur John Bligh, rédacteur en chef de la revue $M$ edical Education, pour la permission de traduire et adapter l'article origi nal. N ous remercions aussi vivement messieurs les docteurs J ean Jouquan (Brest) et $M$ athieu $\mathrm{N}$ endaz ( $\mathrm{G}$ enève) pour leurs préci eux conseils lors de la pré lecture du manuscrit. N ous sommes toutefois seuls responsables de la version finale. $N$ ous remercions aussi nos éudiants, notre rai son d'être comme en seignant, qui, au fil des années, nous ont permis de développer et de raffiner la démarche présentée. A eux tous, merci et bon succès. 


\section{Références}

\section{Tableau 1 \\ Planification d'une étude expérimentale et rédaction \\ d'une demande de subvention en 8 étapes, 28 questions}

\section{ÉT APES / QUESTIONS}

\section{1- QUESTION DE RECHERCHE}

1- Q uel sujet de recherche ou problème vous intéresse?

2- Q uelles recherches ont déjà été effectuées à ce sujet ? Les références.

3- Q uels sont les effets (variable(s) dépendante(s)) qui vous intéressent principalement?

4- Quelle intervention (variable indépendante) vous intéresse?

5- Recherchez-vous des différences ou une association entre variables ?

6- A quel groupe (population) désirez-vous appliquer vos résultats?

7- Q uelle est votre question de recherche?

8- Q uelle réponse attendez-vous ?

Les hypothèses de recherche.

9- Pourquoi cette question est-elle importante? La pertinence.

\section{2- INSTRUMENTS DE MESURE}

10- Utiliserez-vous un instrument de mesure existant, que vous allez modifier ou non, ou en développerez-vous un nouveau?

11- Q uelles sont les qualités psychométriques des mesures (scores) ?

\section{3- PLAN DE RECHERCHE}

12- Voulez-vous intervenir ou simplement observer?

13- Avez-vous besoin d'un groupe contrôle?

14- Comment allez-vous contrôler les variables confondantes?

15- Q uel est le meilleur plan de recherche pour répondre à la question?

\section{4- STATISTIQUES}

16- Q uelle méthode statistique est-elle optimale?

\section{5- ÉCH ANTILLON}

17- Q uels sont les critères d'inclusion et d'exclusion des participants?

18- Comment allez-vous obtenir les participants?

19- Comment allez-vous assigner les participants à leurs groupes?

20- Combien de participants sont-ils nécessaires? Puissance statistique.

\section{6- RECUEIL DES D ONNÉES}

21- Comment allez-vous recueillir les données et contrôler leur qualité?

\section{7- ECHÉANCIERSET BUDGET}

22- Q uels seront les échéanciers?

Les horaires.

23- Q ui fera quoi ? Le personnel.

24- D e quel équipement, de quel matériel aurez-vous besoin ?

25- Q uels seront les coûts ? Le budget.

\section{8- PROTOCOLE ET DEMANDE DE SUBVENTION}

26- Comment allez-vous suivre les progrès de l'étude ? Le protocole.

27- Q uelles sont les priorités de «l'organisme subventionnaire $\gg ?$

28- Q uels formulaires devrez-vous compléter et quelles procédures suivre? 


\section{Tableau 2 \\ Guide de préparation d'une demande de subvention*}

\section{1- PAGE TITRE}

1.1-T Titre de l'éude proposée(voir nombremaximum de frappes permises)

1.2- Chercheur principal : nom, diplôme(s), poste (échelon), département, adresse postale, courriel, tééphone, FAX

1.3- Participants humains: $\mathrm{O}$ ui / $\mathrm{N}$ on

1.4- Approbation du comité d'éthique: O ui / N on / A venir

1.5- Période (dates) de la subvention: de (AA/M M /JJ) à (AA/M M/JJ)

1.6- N ombre total de mois

1.7- Coûts directs et indirects (administratifs) pour la première année de subvention

1.8- Coûts directs et indirects pour la durée totale du projet

1.9- Responsableadministratif s la labvention est accordée: nom et adresse

1.10- Signature du chercheur principal

1.11- Signature du responsable administratif

1.12- Date

\section{2- PAGE RÉSUMÉ}

Format structuré(voir nombre maximum de mots permis):

2.1- 0 bjectifs (question et hypothèses de recherche)

2.2- Contexte et importance

2.3- Plan de recherche (type d'étude)

2.4- Population visée et échantillonnée; échantillon (y compris taille) et méthode d'échantillonnage

2.5- M esure des principaux résultats (variable(s) dépendante(s))

2.6- Intervention (variable indépendante)

2.7- Résultats et conclusions anticipés (voir hypothèses descriptives)

2.8- Échéanciers et personnel

Liste des mots clés ( 6 maximum)

\section{3- PERSONNEL PRINCIPAL}

Liste du personnel principal (chercheur principal, co-chercheurs, assistants de recherche et consultants). Pour chaque personne, indiquez (si applicable) nom, poste (échelon), diplômes (domaine d'étude, institution, lieu, année(s)), certificat (de spécialiste) et droit de pratique, nombre de publications revues par des pairs (y compris 6 références principales), nombre de subventions de recherche (antérieures et courantes, y compris titre, agence subventionnaire, montant, durée et temps consacré).

\section{4-BUDGET DÉTAILLÉ POUR LA PREMIÈRE ANNÉE DE SUBVENTION}

4.1- Personnel : nom, rôle, pourcentage de temps consacré au projet, salairedemandé, bénéfices sociaux, coût total
4.2- Consultant(s)

4.3- Équipement

4.4- $M$ atériel de bureau

4.5- Paiement des participants

4.6- Frais de voyage (liés à la réalisation del'étude et pour présentation des résultatsà des congrès scientifiques)

4.7- Autres dépenses

4.8- Total des coût directs

4.9- Total des coûts indirects (administratifs, si appropriés)

4.10- Coût total (directs et indirects)

5- BUDGET DÉTAILLÉ POUR LA PÉRIODE RESTANTE (voir 4 ci-dessus)

6- JUSTIFICATION DU BUD GET (chaqueitem doit êtrejustifié)

7- RESSO URCES Insallationset équipement, ains que lesoutien local

8- PLAN DE RECHERCHE (voir nombre maximum de pages permises)

8.1- État de la question, buts spécifiques et question(s) de recherche

8.2- Contexte (y compris une analyse critique des références) et importance

8.3- Intervention et mesure des principaux résultats

8.4- Instruments de mesure

8.5- $\mathrm{H}$ ypothèses de recherche

8.6- Plan de recherche (type d'étude) et variables confondantes

8.7- Analyse des données

8.8- Population visée et échantillonnée, échantillon (y compris taille et puissance statistique) et méthode d'échantillonnage 8.9- Recueil des donnéeset contrôles de qualité

8.10- Échéanciers (en mois selon cinq étapes principales) : Préparation, Essais pilotes des instruments de mesure et des procédures, Recueil des données et contrôles de qualité, Analyses des données et $D$ iffusion des résultats (rapport, résumés, articles de revues scientifiques)

\section{9- RÉFÉ REN CES CITÉES (seulement ; pas de bibliographie)}

10- ÉT HIQUE (approbation et formulaire de consentement)

* Préparéen partie (avec autorisation) selon leformulaire des $N$ ational Insitutes of $\mathrm{H}$ ealth (N IH) Application for a Public H ealth Service G rant, O ffice of Extramural Research, United States D epartment of $\mathrm{H}$ ealth and $\mathrm{H}$ uman Services 


\section{Références}

\section{Références}

1. Bordage G, D awson B. Experimental Study D esign and $G$ rant Writing in Eight Stepsand 28 Q uetions. M ed Ed $2003 ; 37$ : 376-85.

2. Bordage G. Reasons Reviewers Rej ect and Accept $M$ anuscripts: The Strengths and Weaknesses in Education Reports Acad M ed 2001; 76 : 889-96.

3. $M$ arks RG, D awson-Saunders $B$, Bailar JC, D an BB, Verran JA. Interactions Between Statisticians and Biomedical Journal Editors. Statistics in M edicine 1988; 7 : 1003-011.

4. Franke JR, Wallen NE. H ow to D esign and Evaluate Research in Education (5th ed.) N ew York: M CGrawHill Higher Education; 2002. (Voir en français Robert $M$. Fondements et étapes de la recherche scientifique en psychologie. St-H yacinthe: EDISEM ; 1988.)

5. Dawson B, Trapp RG. Basic and Clinical Biostatistics (3rd Ed.) N ew York: M c-Graw Hill, Lange M edical Series; 2001.

6. H owell DC. Statistical M ethods for Psychology (5th ed.) Pacific Grove (CA): D uxbury, Wadsworth G roup, Thompson Learning, Inc.; 2002. (Voir en français: Bertrand R. et ValiquetteC. Pratique de l'analyse statis tique des données M ontréal: Presses de l'U niversité du Q uébec, 1986.)

7. M ager R. Preparing Instructional $O$ bjectives (revised 2nd ed.) Belmont (CA): Pitman Learning, Inc.; 1984.

8. Joint Task Force of Academic M edicine and the GEARIM E Committee on Review Criteria for Research $M$ anuscripts ( $G$. Bordage, chair and $A$. Caelleigh, cochair). Acad M ed 2001; 76:897-78.

9. Education Group for Guidelines on Evaluation. Guidelines for evaluating papers on educational interventions. BM J 1999; 318: 1265-7.
10. Application for a Public H ealth Service Grant (PHS 398-Rev. 05/01), NIH Formsand Applications, Office of Extramural Research, $\mathrm{N}$ ational Insitutes of $\mathrm{H}$ ealth (N I H ), U nited States D epartment of $\mathrm{H}$ ealth and Human Services <http://grantsl.nih.gov Ig antstormshtm> Accédéle 7.08.2002.

11. Huth EJ. Writing and Publishing in M edicine ( ${ }^{\text {rd }}$ ed.) Baltimore: Williams and Wilkins; 1999.

12. Bordage G. La préparation d'un artide pour publication. Pédagogie M édicale $2002 ; 3$ : 237-48.

13. H uth EJ. Structured abstracts for papers reporting clinical trials Ann Intern M ed 1987 ; 106 : 626-7.

14. Bordage G, Q uérin S. Le résuméstructuré: un outil de lecture, d'éval uati on et de rédaction. P édagogie M édicale 2001; 2 : 81-4.

15. H enry RC, Wright DE. When D o M edical Students Become H uman Subjects of Research? The Case of Program Evaluation. Acad M ed 2001; 76 : 871-5.

16. Roberts LW, Geppert C, Connor R, N guyen K, Warner TD. An Invitation for M edical Educators to Focus on Ethical and Policy Issues in Research and Scholarly Practice. Acad M ed 2001; 76 : 876-85.

17. M orrison J, Prideaux D. Ethics Approval for Research in M edical Education. M ed Ed 2001; 35 : 1008. 